\title{
La comprensión del movimiento rectilíneo a través de las representaciones semióticas
}

\section{The understanding of rectilinear motion through semiotic representations}

\author{
Nicolle Johana Gómez Rodríguez \\ Didson Harvey Sánchez Monroy² \\ Omaida Sepúlveda Delgado³ \\ Universidad Pedagógica y Tecnológica de \\ Colombia \\ Tunja, Boyacá, Colombia
}

\section{RESUMEN}

Se presenta en el documento una experiencia de aula, resultado de un trabajo de investigación enfocado en exteriorizar el uso de la teoría de las representaciones semióticas para la enseñanza y aprendizaje del movimiento rectilíneo, con el fin analizar las actividades cognitivas de tratamiento y conversión realizadas por los estudiantes con el objetivo de lograr una mejor comprensión del

$1 \quad$ Licenciada en Matemáticas nicolle.gomez@uptc.edu.co

https://orcid.org/0000-0002-1492-6854

2 Licenciado en Matemáticas

didson.sanchez@uptc.edu.co

https://orcid.org/0000-0002-2526-1229

3 Doctora en Educación, Magister en ciencias Matemáticas, Licenciada en Matemáticas

omaida.sepulveda@uptc.edu.co objeto de estudio. En ese sentido, se diseñaron e implementaron situaciones creando condiciones para la realización de los procesos de formación, tratamiento y conversión entre los diferentes registros de representación semiótica, según la teoría de los registros de representación. Para el desarrollo del estudio se realizó la investigación con un enfoque cualitativo siguiendo el diseño de investigación-acción con las etapas de planeación, acción y evaluación.

Como resultado principal se evidencia en el estudio y según los resultados presentados que los estudiantes parten de los conocimientos previos para solucionar cada situación planteada, así como también que se apoyan principalmente en los sistemas de representación 
gráfico y verbal, realizando el respectivo tratamiento y las conversiones entre registros semióticos, promoviendo además el desarrollo del razonamiento matemático a través de la resolución de problemas.

\section{PALABRAS CLAVE}

Representaciones semióticas; movimiento rectilíneo; sistemas de representación; actividades cognitivas; enseñanza; aprendizaje.

\section{ABSTRACT}

The document presents a classroom experience, which is the result of a research work focused on externalizing the use of the theory of semiotic representations for the teaching and learning of rectilinear movement, in order to analyze the cognitive activities of treatment and conversion carried out by the students with the aim of achieving a better understanding of the object of study. In that sense, situations were designed and implemented creating conditions for the realization of the processes of formation, treatment and conversion between the different registers of semiotic representation, according to the theory of the representation registries. For the development of the study, the research was carried out with a qualitative approach following the research-action design with the stages of planning, action and evaluation.

As a main result, it is evident in the study and according to the results presented that students start from the previous knowledge to solve each situation posed, as well as they rely mainly on the systems of graphic and verbal representation, performing the respective treatment and conversions between semiotic registers, promoting furthermore the development of mathematical reasoning through problems solving.

\section{KEYWORDS}

Semiotic representations; rectilinear motion; representation systems; cognitive activities; teaching; learning.

\section{INTRODUCCIÓN}

La enseñanzay el aprendizaje de las matemáticas a lo largo de la historia evidencian diversa clase de dificultades en el aula: en este aspecto, dando una mirada al proceso de la comprensión de los objetos matemáticos, surgió la necesidad de realizar una intervención en el aula para hacer uso de los sistemas de representación de Duval (1999), en un trabajo que involucra las actividades cognitivas de tratamiento $\mathrm{y}$ conversión de las representaciones semióticas para la construcción del objeto del movimiento rectilíneo.

El objetivo de la investigación corresponde a analizar las actividades cognitivas realizadas por los estudiantes para posibilitar una mejor comprensión y desarrollo del aprendizaje de las representaciones semióticas en la solución de situaciones propias del movimiento rectilíneo. Para esto se plantearon situaciones problema en contexto utilizando la teoría de los registros de representación y la resolución de problemas de Mason, Burton y Stacey (1998), esta última, para promover el desarrollo del razonamiento matemático y llevar a los estudiantes al desarrollo de un conocimiento crítico en actividades como interrogar, desafiar y reflexionar sobre la respuesta encontrada; procesos utilizados y el uso de los sistemas de representación para comunicar y dar solución a los problemas diseñados. Con la utilización de representaciones semióticas los estudiantes realizaron las actividades cognitivas referidas por Duval (1999) relacionadas con la formación, el tratamiento y la conversión de los registros de representación semiótica.

Para el desarrollo del estudio, se realizó una investigación con un enfoque cualitativo y una metodología investigación acción al considerar que, "la práctica del docente es un proceso de 
acción y reflexión cooperativa, donde el docente aprende a enseñar, interviene para facilitar y no para imponer ni sustituir la comprensión de los alumnos, así como también, reflexiona sobre su intervención, ejerce y desarrolla su propia comprensión" (Elliott, 1990). Por tanto, se siguieron las etapas de planeación, acción y evaluación propuestas por esta metodología (Elliott, 1990).

Del análisis realizado a la implementación de las situaciones problema para la enseñanza del movimiento rectilíneo, se concluye que las actividades cognitivas de tratamiento $y$ conversión permitieron la construcción del objeto del movimiento rectilíneo, y facilitaron el análisis de las experiencias prácticas al realizar las conversiones entre registros teniendo en cuenta los criterios de congruencia entre representaciones, de tal forma que se construye un significado propio para los objetos matemáticos y físicos trabajados desde el contexto.

\section{MARCO TEÓRICO}

Para el estudio realizado se toma la teoría de los registros de representación semiótica de Duval (1999) con el fin de diseñar y posteriormente analizar cada una de las situaciones problema establecidas para buscando la comprensión del estudiante cuando puede dar un significado propio al objeto matemático de estudio y en relación con el contexto. Esto debido a la importancia que tienen los sistemas de representación en el aprendizaje de las matemáticas, en concordancia con lo que expresa Duval (1999) "las representaciones semióticas no solo son indispensables para fines de comunicación, sino que son necesarias para el desarrollo de la actividad matemática misma" (p. 14).

En la actividad matemática es necesario utilizar los diversos registros de representación para lograr la comprensión del objeto matemático en estudio, para esto se tienen en cuenta los sistemas de representación: verbal, tabular, gráfico, algebraico y numérico. Duval (1999) menciona la existencia de representaciones mentales, es decir, el conjunto de imágenes y conceptos que un individuo puede tener sobre un objeto.

Según Godino (2003) "una característica importante de la actividad matemática es el uso de diversos sistemas de expresión y representación, además del lenguaje natural: variados sistemas de escritura para los números, escrituras algebraicas para expresar relaciones y operaciones, figuras geométricas, gráficos cartesianos, redes, diagramas, esquemas, etc."

Duval (1999) plantea tres criterios al poner en correspondencia los valores que pueden tomar las diferentes variables en un registro de representación semiótica, entendidos como unidades significantes, de modo que dos representaciones son congruentes si cada unidad significante simple se puede asociar con otra unidad significante elemental; si a cada unidad significante elemental de salida, le corresponde una única unidad significante elemental en el registro de llegada; y si hay el mismo orden posible de aprehensión de estas unidades en las dos representaciones.

En este sentido, la teoría de Duval (1999) presenta un gran aporte para el análisis de situaciones prácticas y problemas matemáticos, ya que las representaciones semióticas son inseparables de la obtención y el desarrollo cognitivo, lo que permite la coordinación de sistemas semióticos en el pensamiento científico. Todo esto apoya el análisis de la información extraída para su comprensión, facilitando la escritura con el uso de los diferentes registros de representación semiótica de que se fundamente la situación problema, se analice y pueda ser argumentada coherentemente. 
Al respecto, Mason, Burton y Stacey (1998) aconsejan reconocer $y$ escribir sobre los pensamientos, impresiones e ideas que se encuentran presentes en el razonamiento matemático, tomar apuntes sobre las sensaciones del momento para llegar a favorecer posteriormente la reflexión. Afirma que la esencia del pensamiento matemático está constituida por la particularización y la generalización al enfrentarse a un problema matemático. Esto, permite que los estudiantes se cuestionen y puedan llegar a orientarse en la solución de los problemas que se plantean de manera que puedan interactuar con las representaciones semióticas y así realizar las actividades de tratamiento y conversión (Duval, 1999).

\section{METODOLOGÍA}

Para el proceso de intervención en el aula se sigue el tipo de investigación-acción porque brinda un aporte significativo, cuando se considera la propuesta de Elliott (1990), al afirmar que la práctica del docente es un proceso de acción y de reflexión cooperativa, donde el docente aprende a enseñar y enseña porque aprende, interviene para facilitar y no para imponer ni sustituir la comprensión de los alumnos $\mathrm{y}$, al reflexionar sobre su intervención, ejerce y desarrolla su propia comprensión.

De igual forma, la investigación acción, lleva a indagar al tiempo que se interviene en las prácticas, realizando ciclos repetitivos de análisis para conceptualizar y redefinir el problema las veces que sean necesarias. Se presentan tres fases esenciales de manera cíclica que son: observar, pensar y actuar. En el proceso de estudio se llevaron a cabo las principales acciones de la investigación acción que constan de los siguientes ciclos: identificar la problemática, elaborar el plan, implementarlo, evaluarlo y realizar realimentación (Hernández, 2014).
En este mismo sentido, Hernández (2014) expresa que "La finalidad de la investigaciónacción es comprender y resolver problemáticas específicas de una colectividad vinculada a un grupo". (p.498). Para llevar a cabo esta investigación se siguió el esquema de las etapas como se muestra en la figura 1.

Figura 1. Etapas de investigación-acción

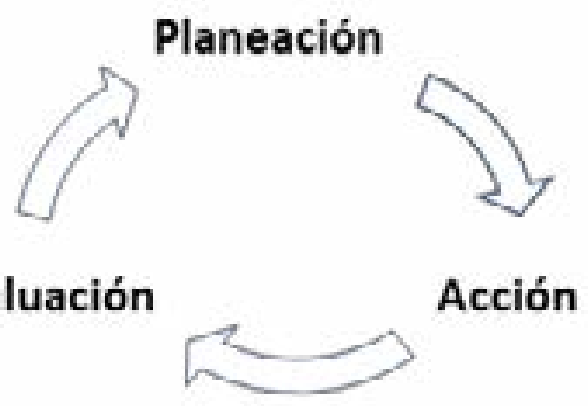

Nota. Adaptado de Elliott (1990)

Las etapas de la investigación-acción se siguieron en el proceso de investigación realizadas con estudiantes de grado décimo de una institución privada de la ciudad de Tunja en el contexto Colombiano. Es pertinente aclarar que este estudio se aplicó a través de una modalidad virtual, utilizando instrumentos para la recolección de datos tales como: grabaciones de video, diario del estudiante, el diario del profesor, foros, pruebas escritas y observación participante.

\section{RESULTADOS}

Según la metodología propuesta y el marco teórico descrito, en la etapa de planeación se diseñaron las situaciones problema, basados en el análisis de experiencias de movimiento rectilíneo con la pretensión de llegar a potenciar el desarrollo del razonamiento matemático con la resolución de problemas del contexto, además de usar las representaciones semióticas para analizar las magnitudes presentes en este. 
En la etapa de acción se realizó la intervención en el aula, en donde se aplicaron las situaciones con los estudiantes de grado décimo, las cuales se enmarcan en los derechos básicos de aprendizaje que contempla la resolución de problemas utilizando propiedades de funciones y usando representaciones tabulares, gráficas y algebraicas para analizar diferentes magnitudes (MEN, 2016). Durante el desarrollo de las situaciones se parte de una prueba preliminar para identificar y reforzar conceptos previos del movimiento rectilíneo, para posteriormente construir el concepto a través de experiencias prácticas y así poderlo aplicar a diversas situaciones problema. Cada tarea se desarrolló en sesiones virtuales, las cuales se llevaron a cabo por medio de la plataforma Google Meet, debido a la actual situación de no presencialidad en los colegios públicos y privados por la emergencia sanitaria, COVID-19.

En la etapa de evaluación se analizó la información recolectada en las grabaciones de la clase, el diario del estudiante, el diario del profesor, los videos, foros y los resultados de las situaciones problema descritos por los estudiantes.
En cuanto al análisis de los instrumentos de recolección de información utilizados en el desarrollo de las situaciones presentadas a los estudiantes de grado décimo para la enseñanza del movimiento rectilíneo, se analizaron los criterios de congruencia entre las representaciones que los estudiantes describen, identificando los aciertos y dificultades presentadas, teniendo en cuenta los aprendizajes fundamentales relativos al razonamiento referidos por Duval (1999).

En la prueba preliminar se observaron algunas dificultades, debido a la no congruencia entre representaciones algebraicas y gráficas, tal y como lo menciona Duval (1999) ya que no hay una correspondencia semántica entre el representante y el representado. Sin embargo, se puede traducir a expresiones que permitan realizar las conversiones adecuadas, tales como la utilización de puntos coordenados para representar valores que luego serán representados en la ecuación particular de cada gráfica o la realización de tablas para poder relacionar directamente la ecuación con la gráfica. Esto se puede observar en la figura 2 con actividades de conversión del registro gráfico al algebraico y viceversa. 
Figura 2. Respuestas de estudiantes E1, E2 Y E3 respectivamente en la tarea 2 de prueba preliminar.

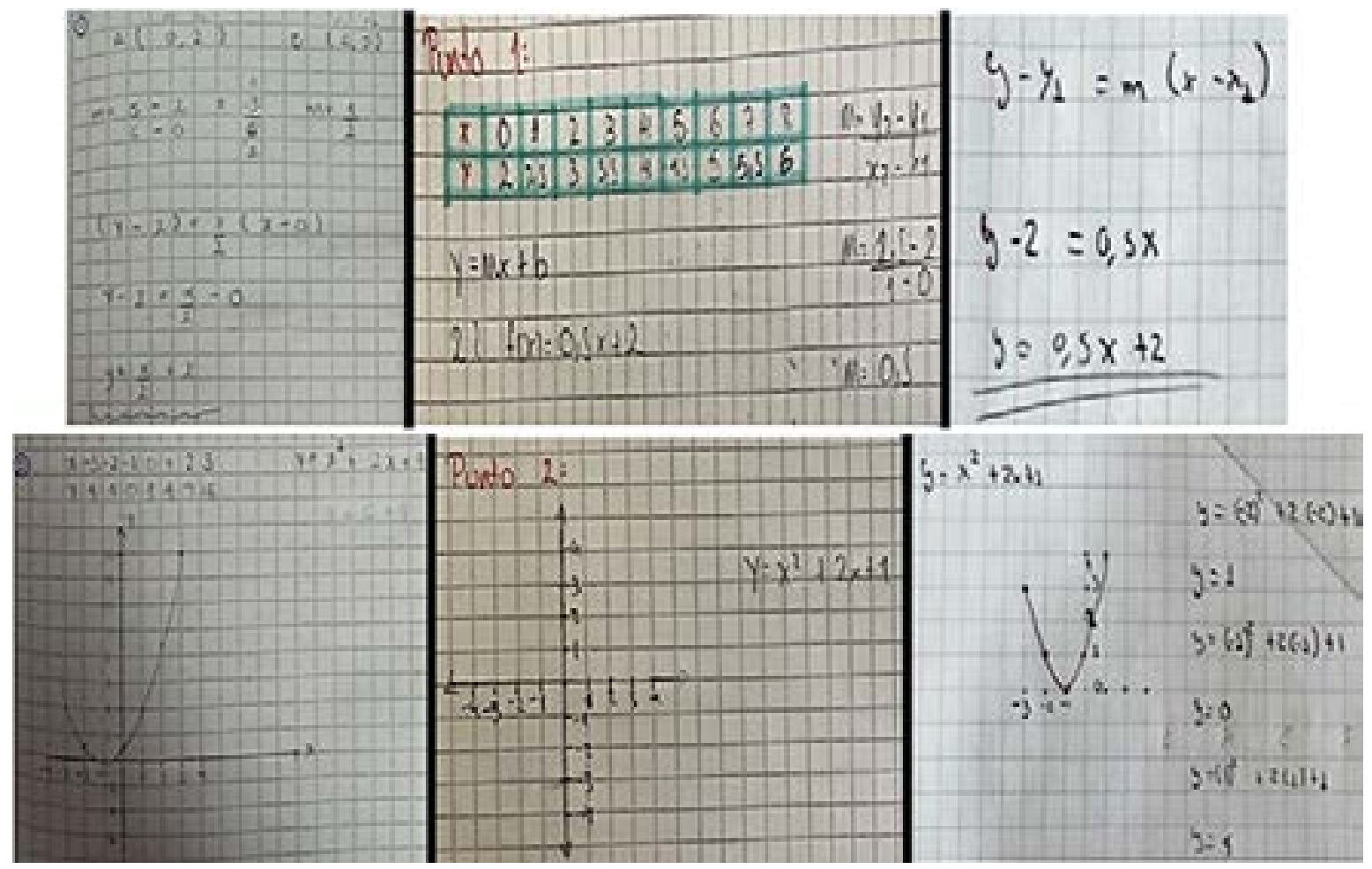

Con la prueba preliminar se puedo observar que los estudiantes realizaron conversiones de registros de representación con mayor facilidad, cuando se encuentra una correspondencia entre éstas, como es el caso de los datos representados en tablas para transformarlos en representación gráfica. También se evidenciaron algunas dificultades en la determinación de los registros adecuados que se utilizan en la conversión, de modo que se concluye que se deben seguir los planteamientos de Duval (2017) respecto al aprendizaje de los estudiantes, respecto a no cuestionar el tipo de registro que se debe elegir, sino actuar y diseñar situaciones donde los estudiantes sean capaces de convertir la representación de cualquier objeto de un registro en otro registro.

Posterior a la prueba preliminar se pasa a realizar la situación problema 1 , con el objetivo de construir el conocimiento del movimiento rectilíneo, de forma que los estudiantes actuaran como matemáticos analizando las diferentes magnitudes encontradas en el movimiento de un cuerpo a través de un objeto que se desplaza por tres trayectos. En este punto, cada estudiante construyó su propio módulo de análisis. En la figura 3 se observa el análisis de uno de los estudiantes donde realiza actividades de tratamiento y conversión con el registro gráfico y tabular basado en los datos obtenidos en la experiencia con el móvil, la cual fue enviada a través de un vídeo por cada estudiante. 
Figura 3. Análisis del estudiante E6 de dos trayectos de la situación problema 1

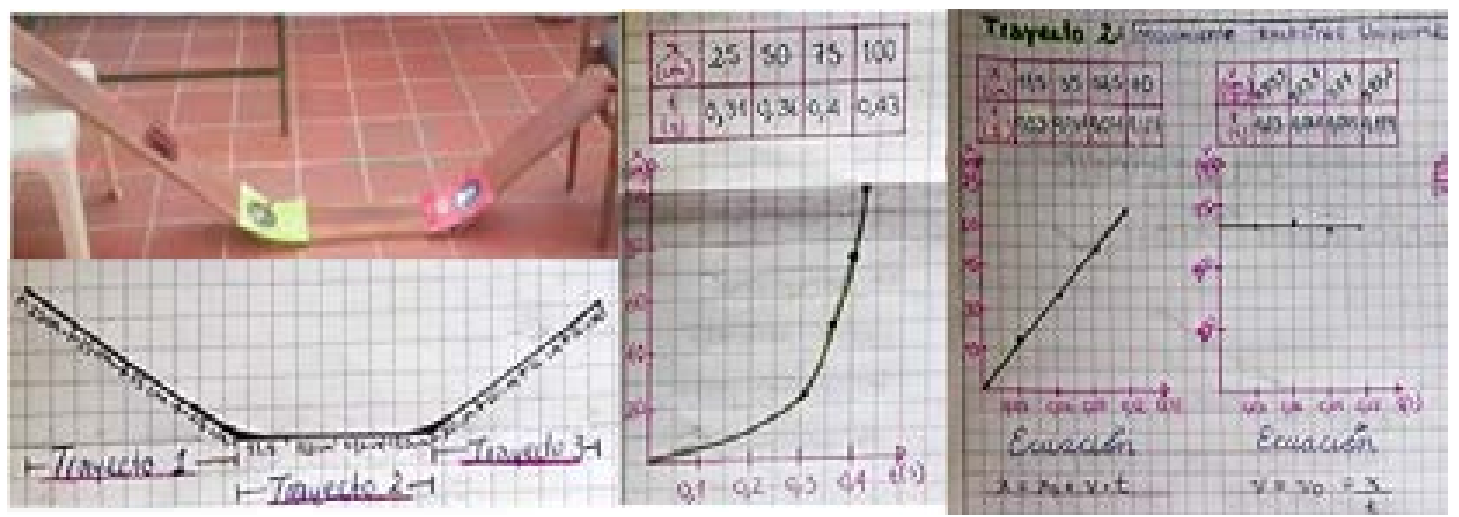

En esta situación problema, los estudiantes desarrollaron ideas básicas del pensamiento matemático como lo plantea Mason et. al. (1998) dando como resultado el descubrimiento y desarrollo del conocimiento del movimiento rectilíneo, de forma que se fueron plasmando diferentes consideraciones del problema en un diario. Con las descripciones realizadas por los estudiantes tanto en el informe como en el diario fue posible evidenciar que le dieron un significado social al objeto de estudio al realizar prácticas conceptuales construyendo cognitivamente conceptos matemáticos con las magnitudes manipuladas (D' Amore et al., 2017). Lo anterior se reflejó en el registro verbal utilizado por los estudiantes, aunque algunos pasaban por alto las unidades significantes correspondientes a algunos aspectos del movimiento.

Se realizó la situación problema 2, con tareas que incluían realizar actividades de transformación de representaciones semióticas teniendo en cuenta los criterios de congruencia al identificar las unidades significantes de cada representación. En general con la situación problema se observaron dificultades para la conversión de representaciones, en particular cuando presentaban fenómenos de no congruencia, el tratamiento del registro presentó falencias, además de la conversión a este, particularmente, desde el registro verbal, ya que en ocasiones no se identificaron las unidades significantes que se encontraban de manera implícita, tales como el tipo de movimiento rectilíneo presentado en la situación problema. Esto es posible observarlo en la figura 4, donde el estudiante realiza una conversión adecuada al registro verbal, pero falla en la conversión al registro gráfico de la distancia con respecto al tiempo.

Figura 4.

Conversión entre registros de representación gráfico y verbal del estudiante E6 para la tarea 1 situación 2.

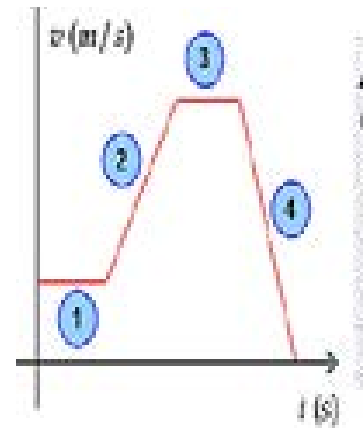

La grática veluobled tienos se cempeith de morena

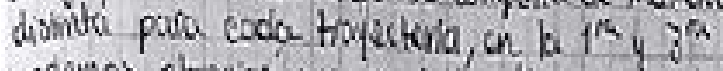
pedemos closicat cue ie trate de nevimilewe jectiline unkerme, in la $2^{\text {to }}$ movimiento ectilindo

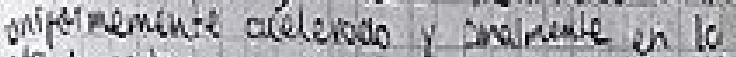

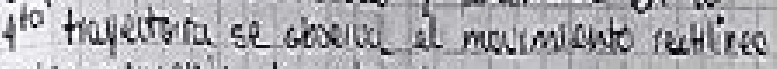
unifsimimens descreleicio.

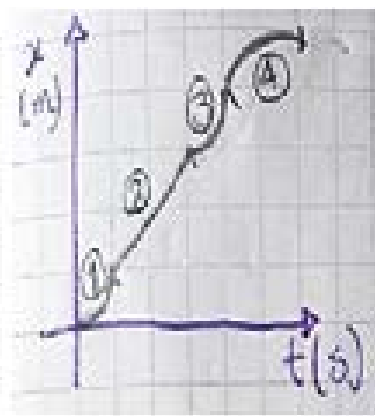


En el desarrollo de la situación problema 2 se pudo observar que los estudiantes utilizan los fenómenos relativos a la semiósis como describe Duval (1999) ya que utilizaron una diversificación de los registros de representación semiótica, para dar solución a la pregunta planteada, los cuales pueden guiar el proceso para encontrar la posible respuesta al problema, además de comunicar sus análisis con diferentes registros tal y como se observa en la figura 5 .

Figura 5.

Conversión entre registros de representación gráfico algebraico y numérico del estudiante E5 para la tarea 4 situación 2.

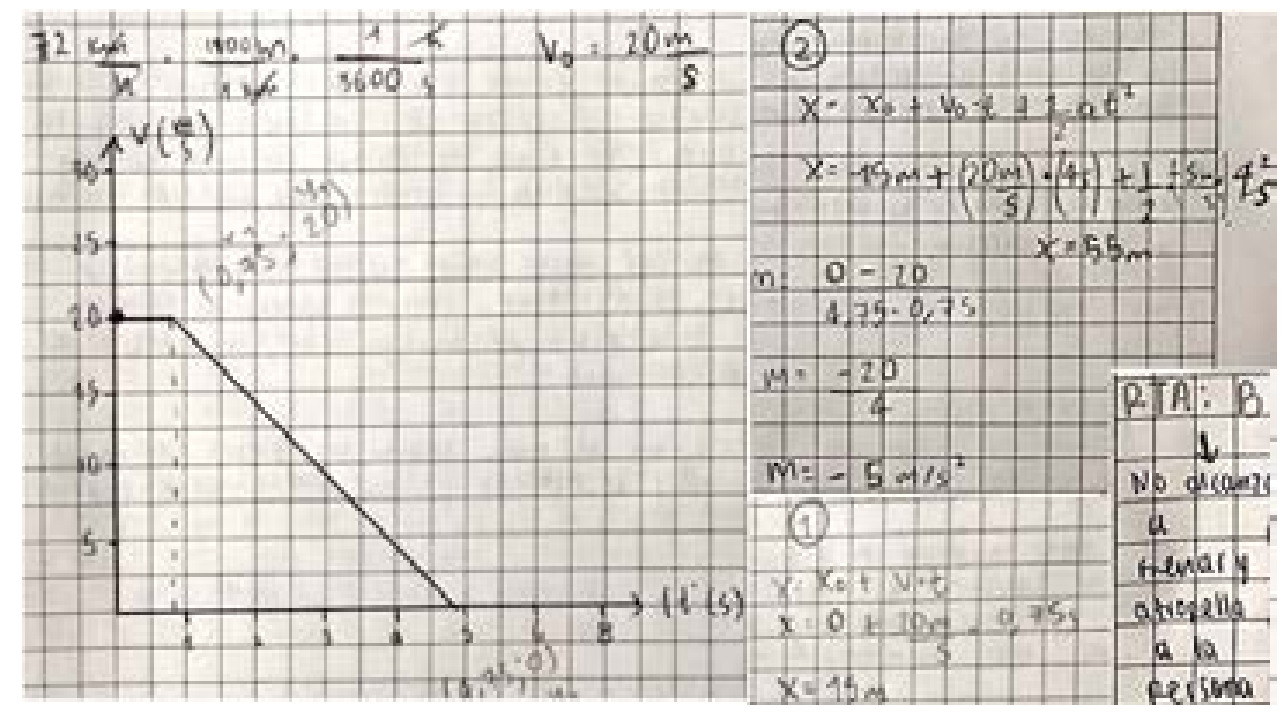

Finalmente, se realizó una tercera situación problema que constaba de 2 experiencias prácticas con objetos en caída libre, de manera que se comunicaran resultados y conclusiones basados no solo en la observación y toma de datos del tiempo, sino también en las representaciones semióticas utilizadas y su relación con la visualización del movimiento a través de simuladores. Con esta situación se pudo observar el uso de diversos registros de representación, sin embargo, en la presentación de conclusiones se omitían algunas unidades significantes en la conversión al registro verbal, teniendo en cuenta los análisis realizados con los diferentes registros de representación.

En la primera tarea de esta situación se analizó la caída libre de un objeto sobre un vaso con agua: los estudiantes presentaron un informe sobre los resultados obtenidos, realizando el tratamiento del registro algebraico para obtener la ecuación de distancia y velocidad con respecto al tiempo y realizando las conversiones al registro gráfico, pasando por el tabular, sin embargo en la descripción verbal de la observación, omitieron algunas unidades significantes tales como la distancia inicial, el tipo de movimiento el cual presenta aceleración, la distancia recorrida, el aumento de velocidad, entre otras, basando sus descripciones en solo algunos aspectos que componen el movimiento. En la figura 6 se puede observar un ejemplo de lo mencionado anteriormente, en donde el estudiante realiza la descripción del movimiento a partir del video realizado por sí mismo. 
Figura 6. Capturas de vídeo y representaciones del estudiante E4, tarea 1 situación 3.

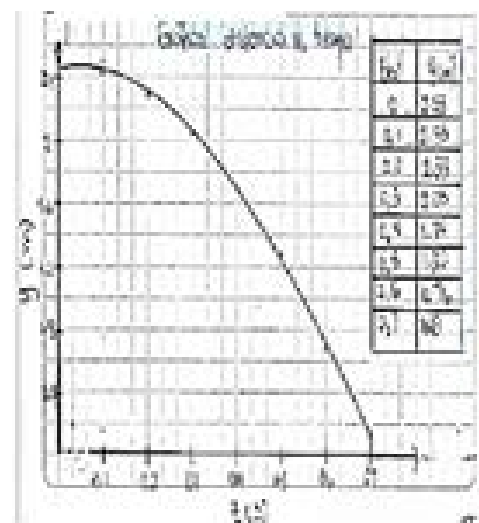

Para ampliar el argumento basado en la observación y en los diferentes registros utilizados, es posible tener presente la incorporación de las nuevas tecnologías ya que como lo menciona Goldin y Shteingold (2001), por medio de ellas, es posible desarrollar sistemas internos de representación, de manera que se pueda visualizar mucho mejor el comportamiento de las magnitudes presentes en el movimiento y además considerar algunos factores como la densidad en el cambio del medio, del aire al agua, de esta manera pueden incorporarse diferentes sistemas de representación que permiten que los estudiantes se apropien de los conceptos y argumenten mucho mejor externalizando sus representaciones internas.

Se concluye también, que el uso de simuladores apoyó en gran medida el análisis y comprensión de la experiencia práctica, evidenciando una relación con los resultados obtenidos. En la tarea 2 de la situación problema 3 , se lanzó un objeto verticalmente hacia arriba y se realizó su respectivo análisis de manera que se compararon valores, como para el caso de la conversión al registro gráfico, tal y como se observa en la figura 7.

Figura 7.

Gráfica distancia tiempo realizado por el estudiante E1 y su comparación con el simulador de movimiento de un proyectil, para la tarea 2, situación 3.

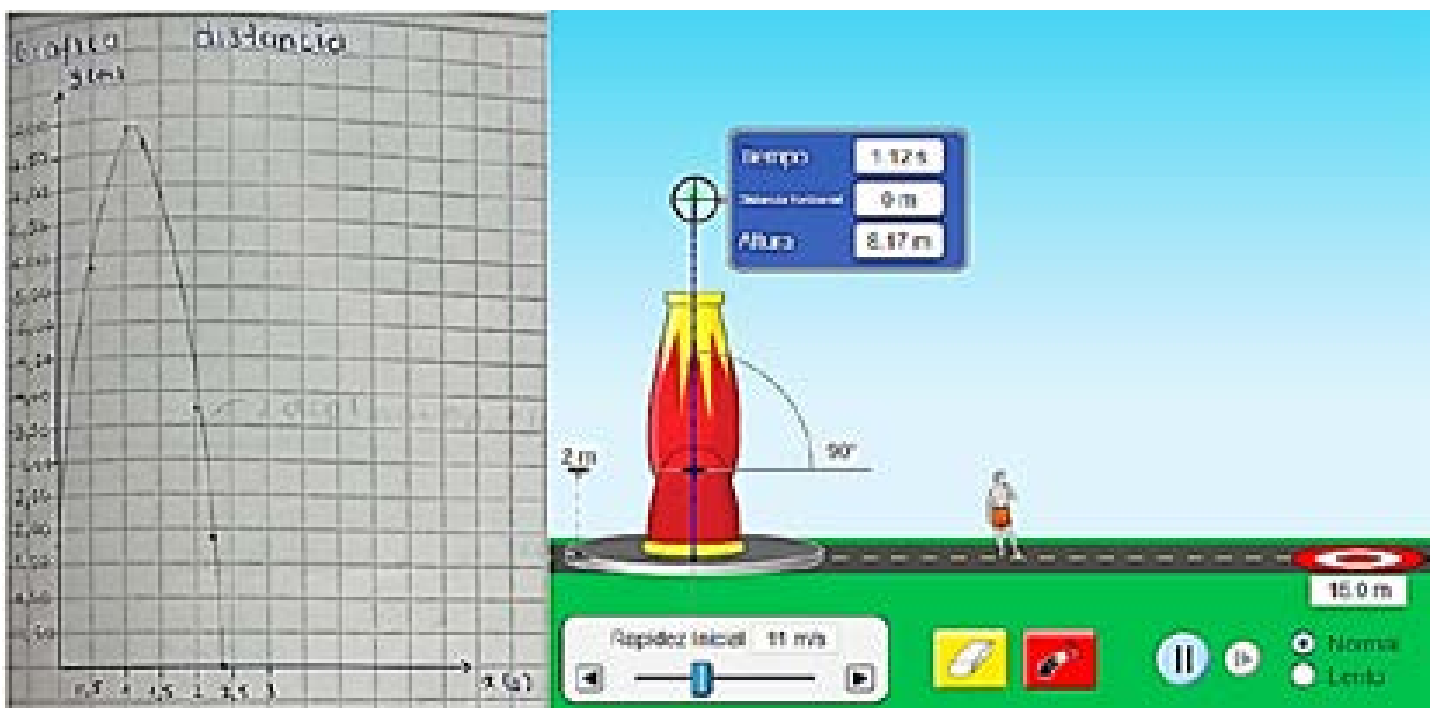

Fuente: Simulador movimiento de un proyectil. Tomado de: https://phet.colorado.edu/sims/ html/projectile-motion/latest/projectile-motion_es.html 


\section{CONCLUSIONES}

Con el uso de las representaciones semióticas, fue posible la construcción del concepto de movimiento rectilíneo, de manera que se coordinaron los registros de representación para el análisis de contextos reales lo cual lleva a establecer un significado propio a los conocimientos adquiridos.

En las situaciones abordadas por los estudiantes, se evidenciaron dificultades en la actividad de conversión de los registros de representación cuando se presentaban fenómenos de nocongruencia entre representaciones como en el caso de la conversión desde el registro verbal al gráfico, al no identificar algunas unidades significantes implícitas en algunas descripciones, sin embargo al extraer los datos suficientes fue posible relacionarlos con los diversos registros de representación para analizar mejor cada situación problema planteado.

Finalmente, se concluye que las situaciones de enseñanza permitieron a los estudiantes construir conceptos basados en sus conocimientos previos para luego movilizarse entre los diferentes registros de representación y así analizar diversas situaciones problema incluyendo un entorno social, de manera que les da un significado propio a los conceptos trabajados. Lo anterior se pudo observar por medio de las representaciones externas realizadas por los estudiantes y sus consideraciones descritas con el registro verbal en los informes y diarios realizados.

\section{REFERENCIAS}

Duval, R. (1999). Registros de representación, comprensión y aprendizaje. En, Semiosis y pensamiento humano ( $p p$. 25-80). La Gaceta de la Real Sociedad Matemática Española.

Duval, R. (2017). Understanding the Mathematical Way of Thinking - The Registers of Semiotic Representations. Dunkerque, France. Université du Littoral Côte d'Opale.

D'Amore, B. (2004). Conceptualización, registros de representaciones semióticas y noética: interacciones constructivistas en el aprendizaje de los conceptos matemáticos e hipótesis sobre algunos factores que inhiben la devolución. Uno. Barcelona, España. 35, 90-106

Elliott, J. (1990). La investigación-acción en educación. Ediciones Morata.

Godino, J. D. (2003). Teoría de las funciones semióticas. Un Enfoque OntológicoSemiótico de la Cognición e Instrucción Matemática, Granada. Universidad de Granada.

Goldin, G., \& Shteingold, N. (2001). Systems of representations and the development of mathematical concepts. The roles of representation in school mathematics, 1-23.

Hernández, R., Fernández, C., y Baptista, P. (2014). Metodología de la investigación Mc Graw Hill. Interamericana Editores.

Mason J., Burton L. y Stacey K. (1988) Pensar Matemáticamente. Editorial Labor, S. A. Madrid, España.

Ministerio de Educación Nacional (MEN). (2006). Estándares Básicos de Competencias en Matemáticas. En, Estándares Básicos de Competencias en Lenguaje, Matemáticas, Ciencias y Ciudadanas. (pp.46-95). MEN. 